\title{
Effect of Car Rear Shape on Pollution Dispersion in Near Wake Region
}

\author{
Xing-Jun Hu, Han-Bo Yang, Bo Yang, Xiu-Cheng Li, and Yu-Long Lei \\ State Key Laboratory of Automotive Simulation and Control, Jilin University, Changchun, Jilin Province 130022, China \\ Correspondence should be addressed to Yu-Long Lei; leiyl@jlu.edu.cn
}

Received 19 March 2015; Revised 22 June 2015; Accepted 13 July 2015

Academic Editor: Francesco Braghin

Copyright (C) 2015 Xing-Jun Hu et al. This is an open access article distributed under the Creative Commons Attribution License, which permits unrestricted use, distribution, and reproduction in any medium, provided the original work is properly cited.

\begin{abstract}
Dispersion of vehicle exhaust gas is a primary source of air pollution in urban areas. Thus, it has become an important subject in the automotive field. This paper consists of two parts. First, the fastback MIRA model was selected as study object and a standard $\kappa-\varepsilon$ two-equation turbulence model was used. The simulation results were compared and analyzed with experimental data. The feasibility of the turbulence model and grid strategy was then verified, and the results were used in the next research step. Second, we used propane as tracer gas while ignoring the effect of the vehicle wake field force on this gas. The tracer gas diffusion in the wake flow field was then simulated through fastback, notchback, and square-back MIRA models. This study focuses on analyzing the characteristics of wake field diffusion effects, particularly in the near wake of a vehicle.
\end{abstract}

\section{Introduction}

The term " $\mathrm{PM}_{2.5}$ " refers to micron particles that have an aerodynamic diameter of less than or equal to $2.5 \mu \mathrm{m}$ in the atmosphere. These particles mainly originate from residues produced in the process of daily power generation, industrial production, and vehicle emissions. Thus, these particles contain toxic components such as heavy metals [1].

Dispersion of vehicle exhaust gas is a major cause of air pollution in urban areas. Studying the dispersion behavior of vehicle exhaust gas is significant to improve living quality and inhabitation environment, as well as to achieve sustainable development [2].

In this research field, CFD technology has many advantages, such as low cost of money and time, ease in changing boundary conditions, and high repeatability. Foreign scholars have studied diffusion characteristics using the CFD technique, such as simple exhaust diffusion model [3], dispersion of vehicle pollutants under idle condition $[4,5]$, different driving conditions [6], vehicle queues [7], and effect of bodytail modeling on the diffusion properties [8].

Nucleation, condensation, coagulation, deposition, and other phenomena [9-11] during the pollution diffusion process are ignored. Tracer gas is used in CFD simulation analysis $[8,12]$ to qualitatively examine the exhaust diffusion in the wake flow field of different models. This gas was selected as the tracer gas [12] because of the stable physical nature of propane $\left(\mathrm{C}_{3} \mathrm{H}_{8}\right)$ and its low-volume fraction in the environment.

\section{Simulation Models}

We used 33\% scale MIRA models, including fastback, notchback, and square-back models, in the present study. We slightly modified the size of the fastback model to compare the numerical simulation results with existing experimental data [6], as shown in Figure 1. The vehicle model and application of the coordinate system based on the size of the reference model are shown in Figure 2, where $L$ is the model length, $h$ is the model height, and $b$ is half the width of the model base.

\section{Numerical Simulation}

3.1. Calculation Domain. Previous simulations show that the calculation domain was a large cuboid, the distance from the left and right sides to the field symmetry was 3.5 times 


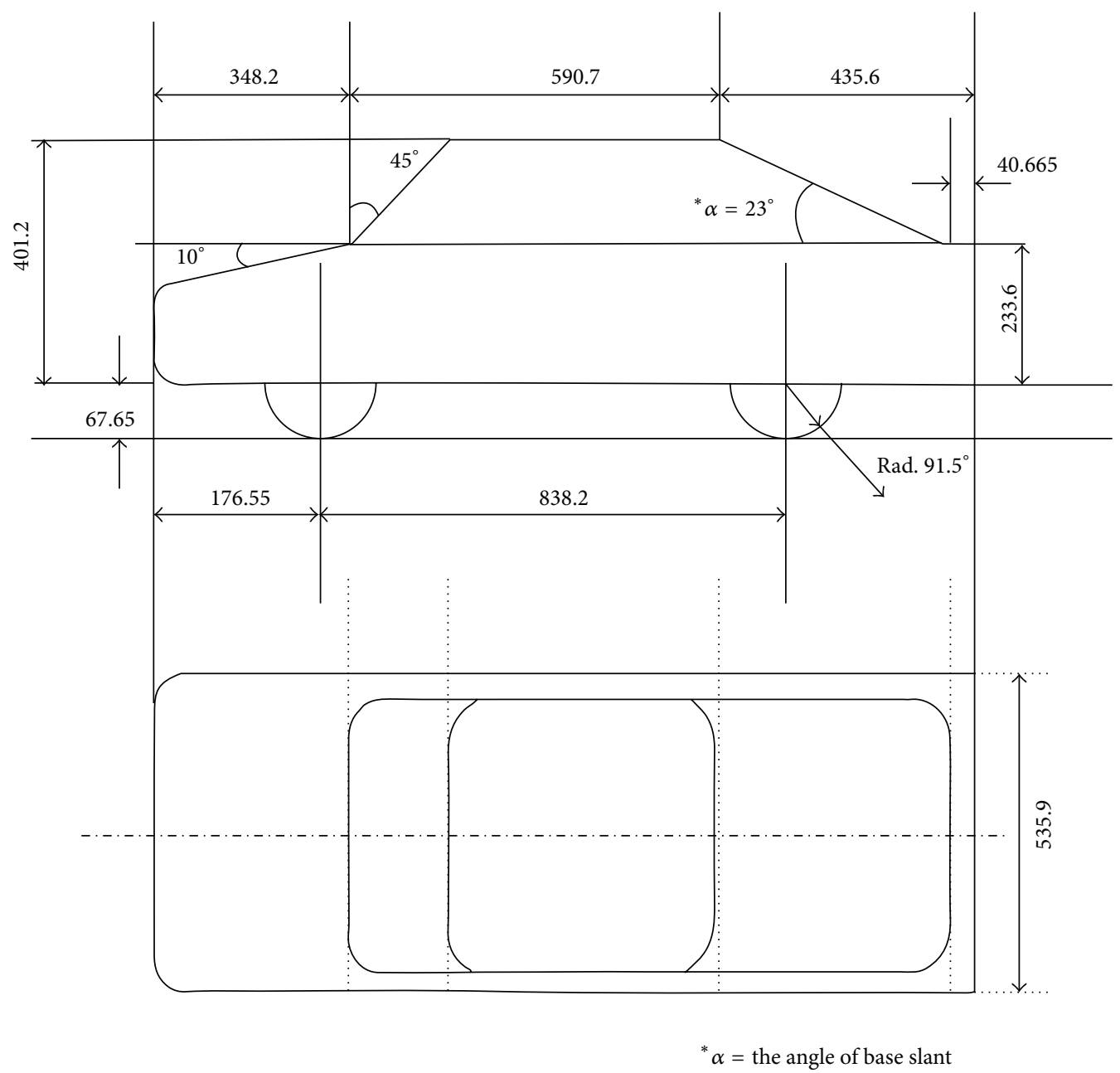

FIGURE 1: 33\% scale MIRA reference model (unit: $\mathrm{mm}$ ).

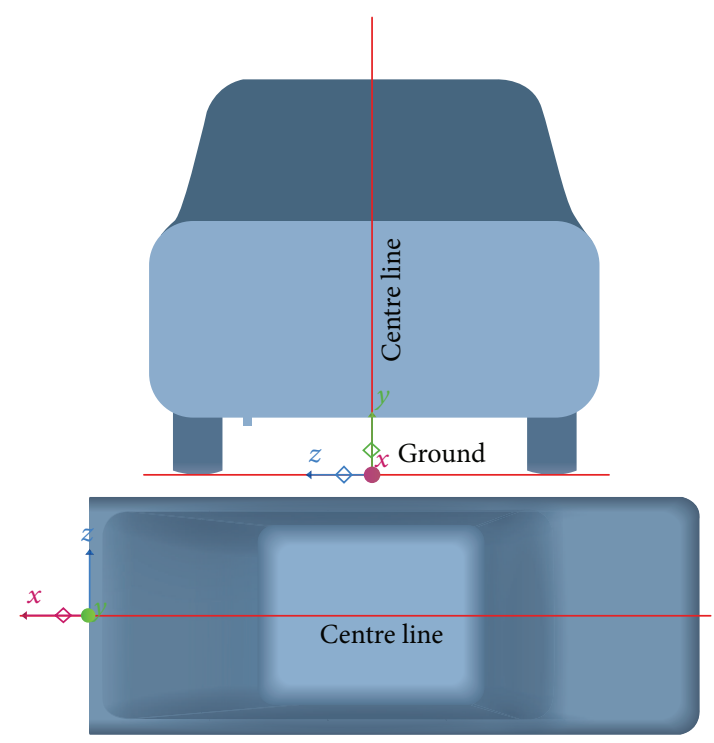

Figure 2: Vehicle model and coordinate system. 


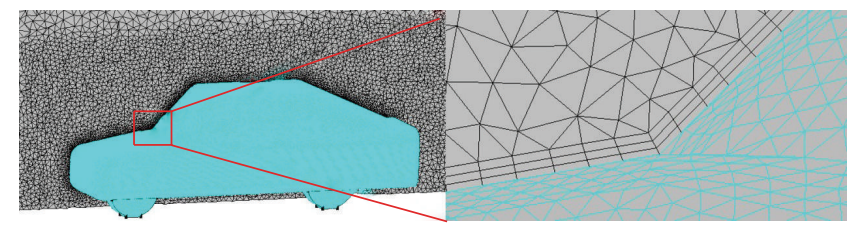

FIgURE 3: Mesh distribution.
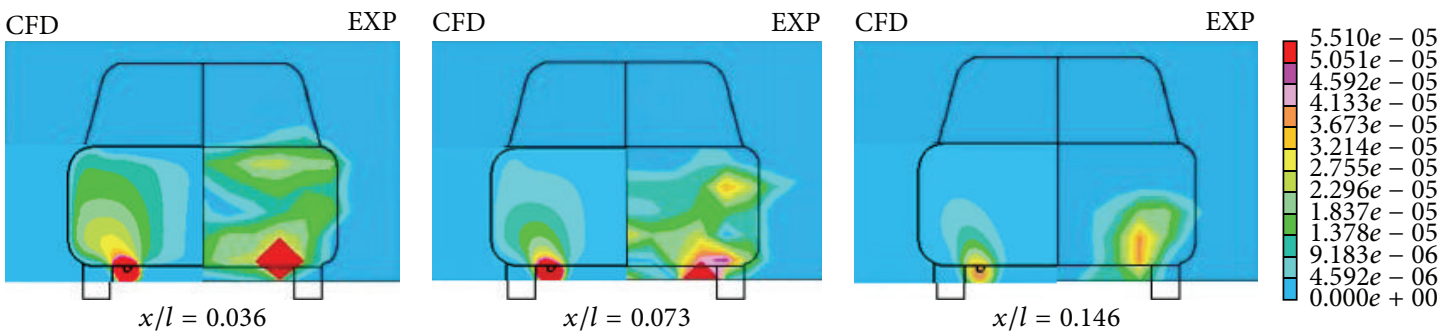

Figure 4: Propane concentration distribution of $x / l$.

the vehicle width, the computational field height was 5 times the height of the vehicle, and the length was 11 times the length of the vehicle 3 times the length of the front part of the vehicle.

3.2. Mesh Strategy. A combination of tetrahedrons and triangular prisms were used in this study. The prisms were divided into three layers to effectively simulate the boundary layer flow. The grid around the body was refined to accurately capture the flow field near the body. The final grid is shown in Figure 3.

3.3. Turbulence Model. The standard $\kappa-\varepsilon$ two-equation turbulence model was adopted, and the finite volume method was used to discretize the governing equations. Simulation analysis was conducted based on mass conservation, momentum conservation, turbulent kinetic energy, diffusive species equations, turbulent dissipation rate equations, and energy equation [13].

The diffusion conservation equation is expressed as follows:

$$
\frac{\partial \rho C}{\partial t}+\frac{\partial \rho C u_{j}}{\partial x_{j}}=\frac{\partial}{\partial x_{j}} \rho D_{m} \frac{\partial C}{\partial x_{j}}+\rho \dot{d} .
$$

In (1), $\rho$ is the density of a fluid or a solid, $x_{j}$ stands for the coordinates, $u_{j}$ is the velocity of flow in $x_{j}$ direction, $\dot{d}$ is the source term of diffusive species, $C$ is the concentration of a diffusive species, and $D_{m}$ is the diffusion coefficient.

\section{Result Analysis and Discussion}

4.1. Numerical Method Validation. The numerical simulation results of the $33 \%$ scale fastback model compared with existing experimental results are shown in Figure 4. The propane concentration distribution and diffusion tendency in the different $x / l$ sections are consistent with the experimental data. Considering that this study performs qualitative research on exhaust diffusion in various models of wake flow fields, we determined that the CFD simulation results were valid. Thus, the mesh strategy and boundary conditions can be used in a subsequent study.

\subsection{Comparison of Three Model Concentration Fields}

4.2.1. Comparison of $z / b=0.5612$ Section. The flow that passes through the bottom of the body entered the upwash region (Figure 5) as a result of the tail inclination in the square-back and notchback models. The exhaust gas diffused upward after being affected by the flow, as shown in Figure 6. At the same time, the exhaust gas entered the low-speed region behind the car because of the influence of the clockwise vortex. The low-speed area of the square-back was larger than the low-speed area of the notchback, so the exhaust gas diffusion area was also larger. This condition is illustrated in the first two pictures in Figure 6.

Comparatively, almost no airflow occurred in the upwash region and the exhaust gas diffused backward directly because the fastback model had no tail inclination. A small amount of exhaust gas entered the low-speed region. The exhaust gas diffusion distance was longer than that of the notchback model of the same height.

4.2.2. Comparison of $y / h$ Sections. The $y / h$ sections crossed with the exhaust pipe are discussed in this part of the paper. The sections of the square-back and notchback models are represented as $y / h=0.2722$, and the section of the fastback model is $y / h=0.0796$.

As shown in Figure 7, a high-speed stream occurred in the wake region of the square-back model. The distribution of the concentration formed a clockwise structure when the stream flowed into the low-speed wake region, as shown in the first picture of Figure 8. Comparing Figures 7 and 8 proves the dependency of diffusing the concentration field on the wake flow field.

In analyzing the concentration field on the $z / b=$ 0.5612 section (Figure 5), we determined that the exhaust of 

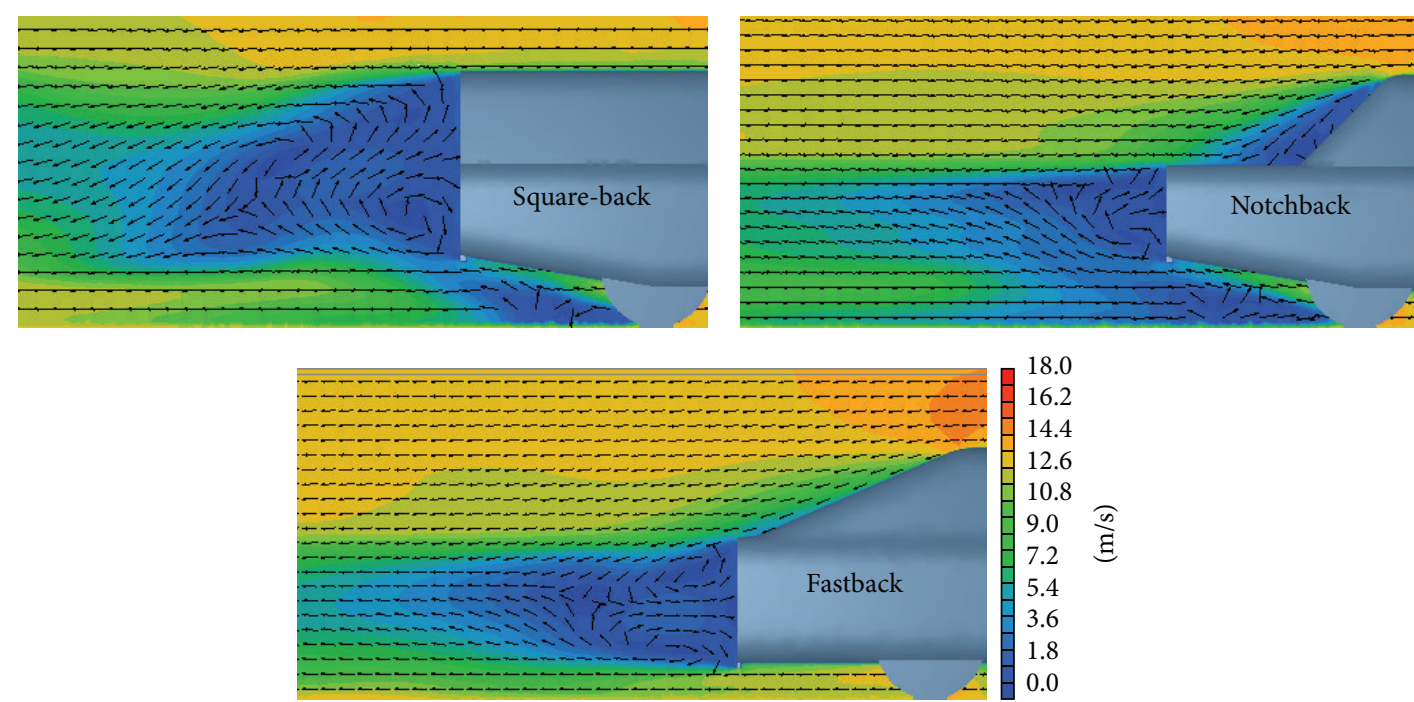

FIGURE 5: Velocity vector of $z / b=0.5612$.
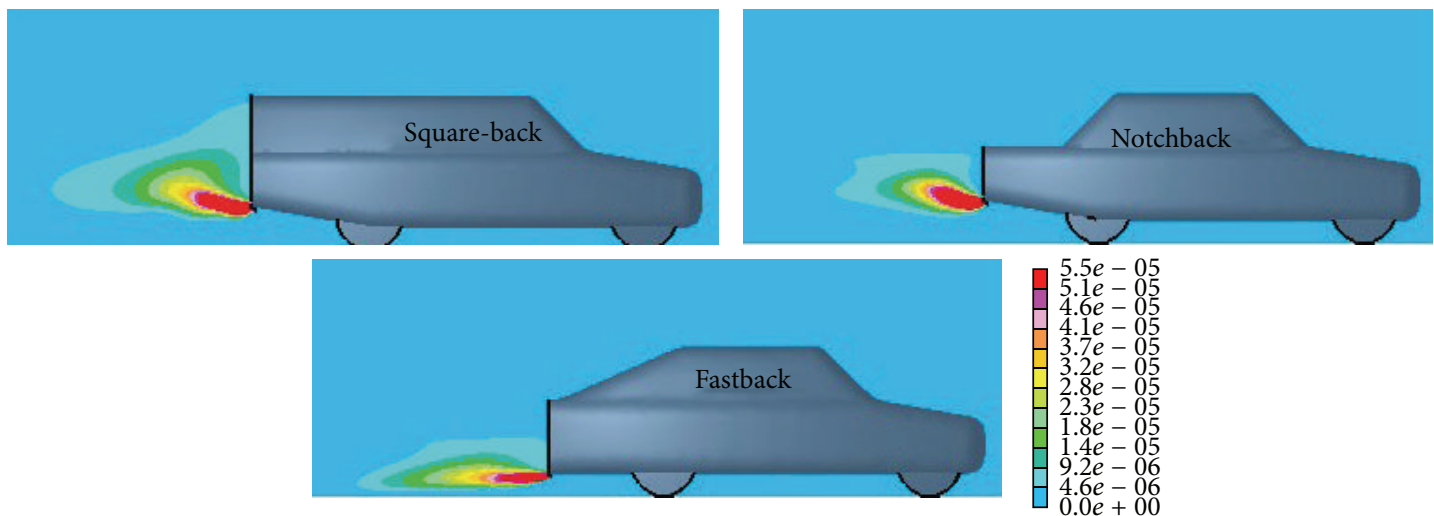

FIgURE 6: Propane concentration distribution of $z / b=0.5612$.
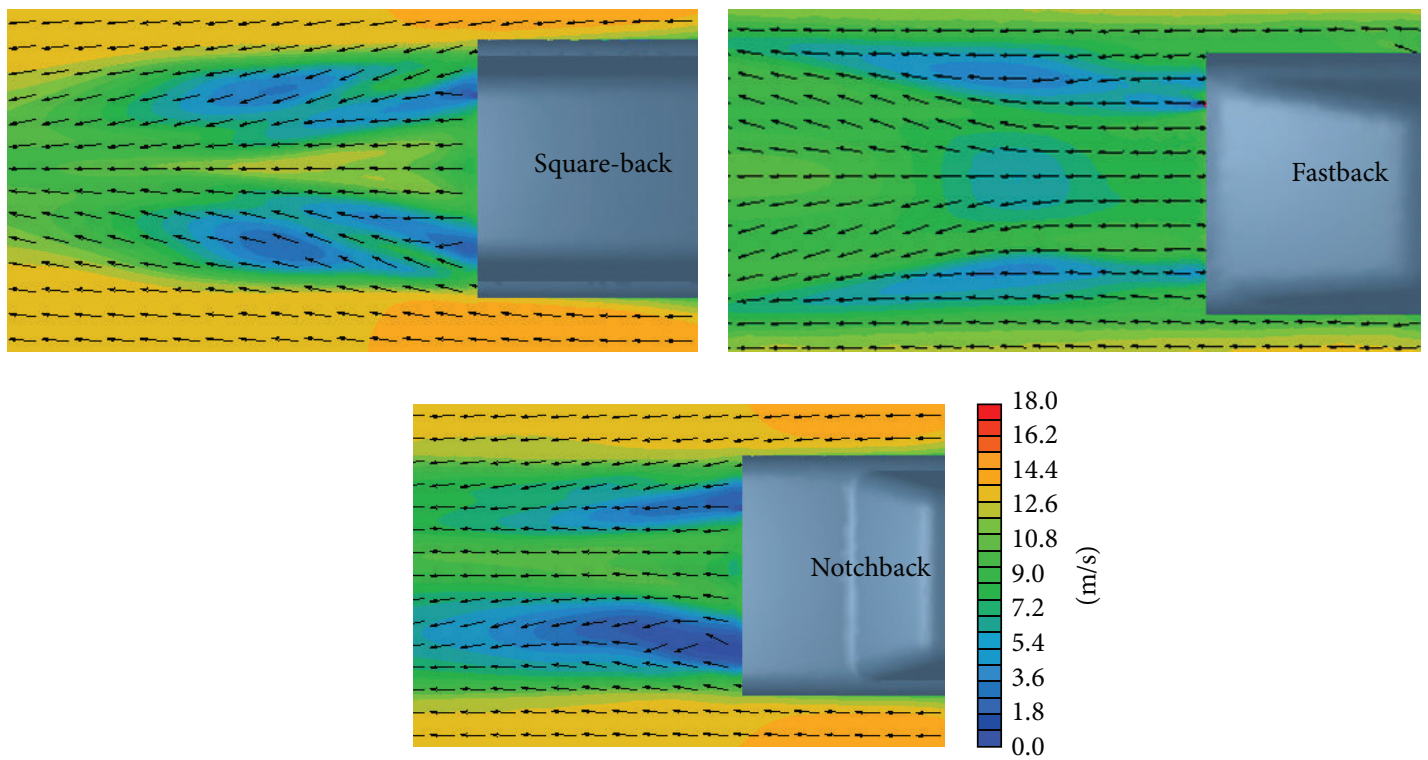

FIgURE 7: Velocity vector on the $y / h$ section. 

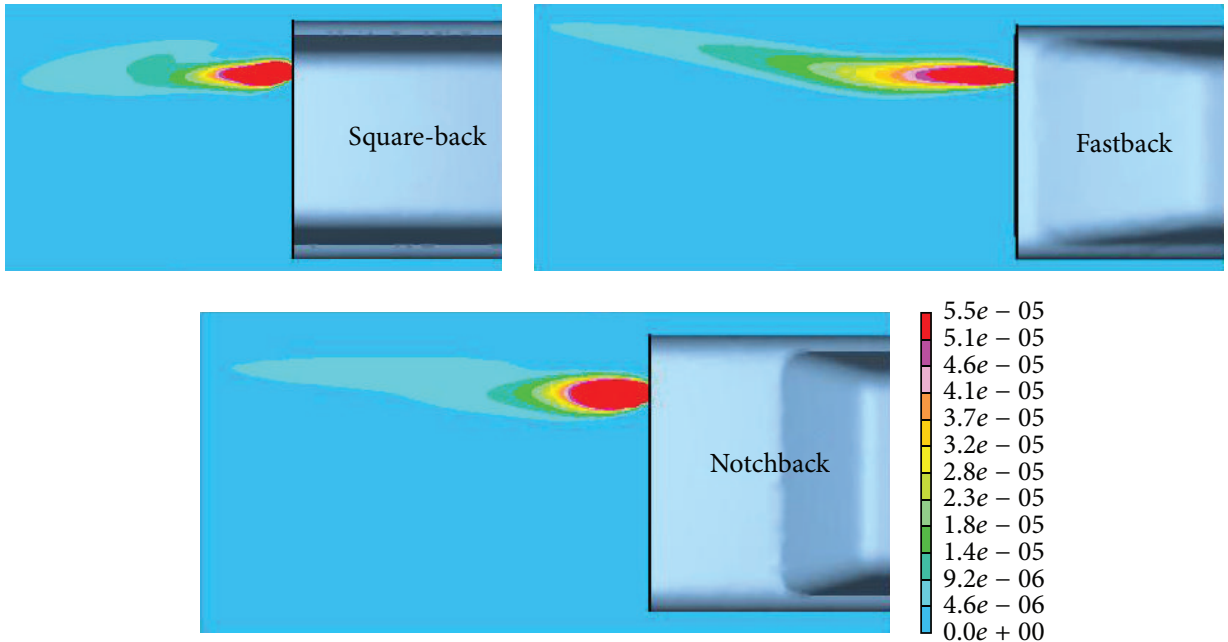

$5.5 e-05$

$5.1 e-05$

$4.6 e-05$

$4.1 e-05$

$3.7 e-05$
$3.2 e-05$

$2.8 e-05$

$2.3 e-05$

$1.8 e-05$

$1.4 e-05$

$9.2 e-06$

$4.6 e-06$

$0.0 e+00$

Figure 8: Propane concentration distribution of $y / h$.

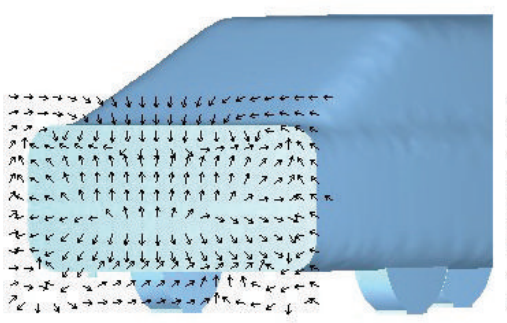

Fastback model

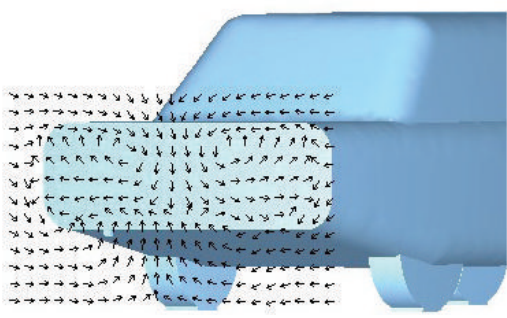

Notchback model

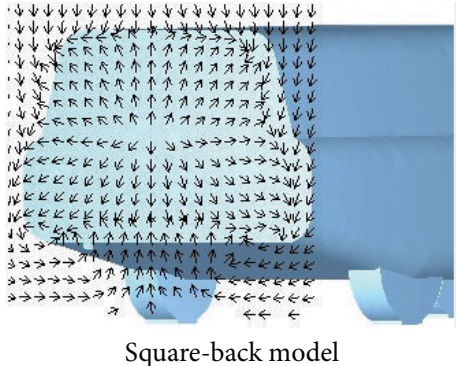

Square-back model

FIgURE 9: Velocity vector on $x / l=0.036$ section.

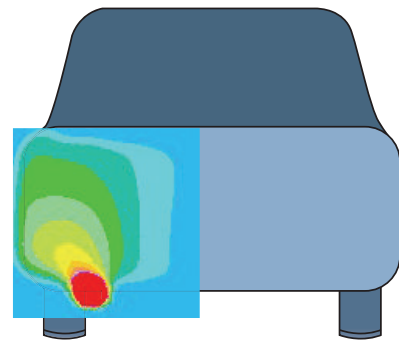

Fastback model

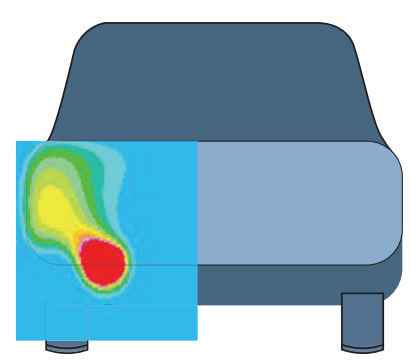

Notchback model

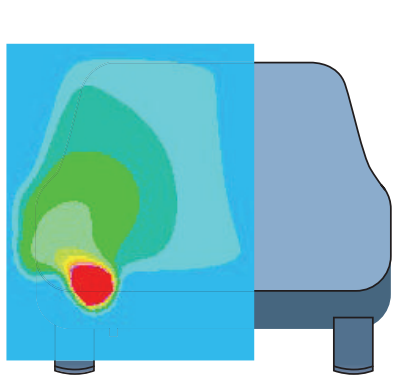

Square-back model
$5.510 e-05$ $5.051 e-05$ $4.592 e-05$

$4.133 e-05$

$3.673 e-05$

$3.214 e-05$

$2.755 e-05$

$2.296 e-05$

$1.837 e-05$

$1.378 e-05$

$-9.183 e-06$

$4.592 e-06$

$0.000 e+00$

FIgURE 10: Propane concentration distribution of $x / l=0.036$.

the square-back and notchback models went up into the lowspeed region. However, the exhaust of the fastback model had minimal changes in the flow direction and was then dragged backward by the airflow. Thus, the amount of exhaust gas that flowed into the low-speed wake region was the least among the three models. Figure 8 shows that the exhaust gas in the fastback model has the longest diffusion distance.

The airflow moved from the center to the side in the $y / h=$ 0.0796 section in the fastback model, as shown in the third picture of Figure 7 . The propane $\left(\mathrm{C}_{3} \mathrm{H}_{8}\right)$ was also diffused outward as indicated by the concentration field in Figure 8.
The other two models show that the diffusion tendency of the concentration field coincided with the corresponding velocity field of the wake flow.

4.2.3. Comparison of $x / l=0.036$ Section. The distributions of propane concentration in the $x / l=0.036$ section of the three models are shown in Figure 10. This figure illustrates that the propane concentration was mainly diffused outward from the autobody in the horizontal direction and upward in the vertical direction. The reason for this condition is shown in Figure 9, which expresses the velocity vector in 


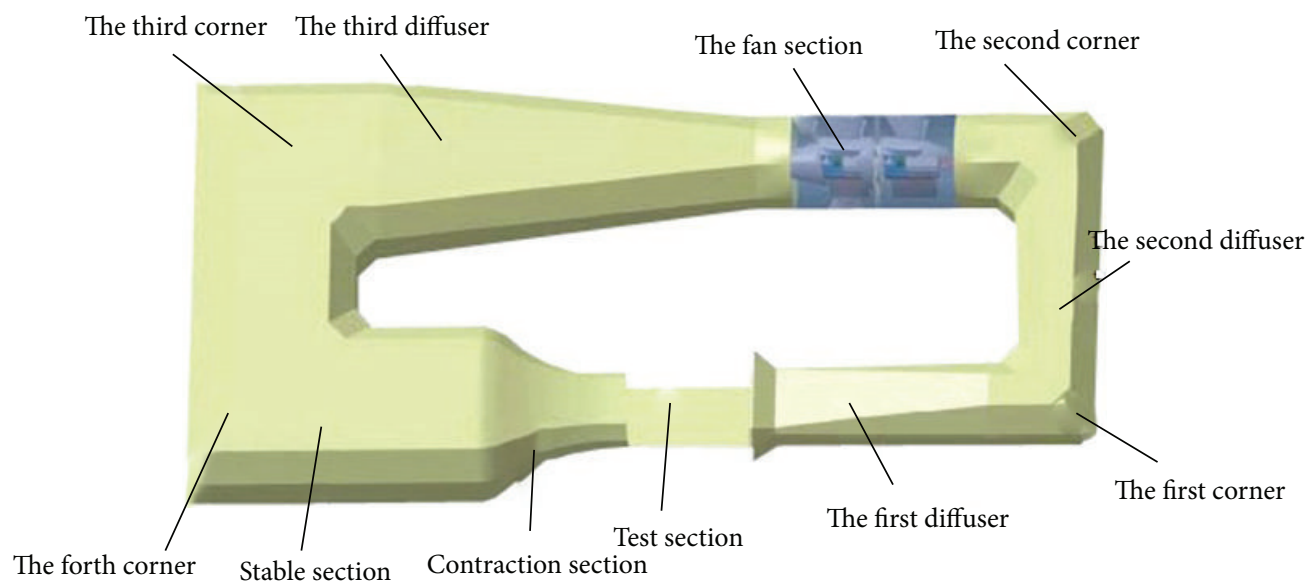

FIgURE 11: The tunnel structure diagram of Jilin University.

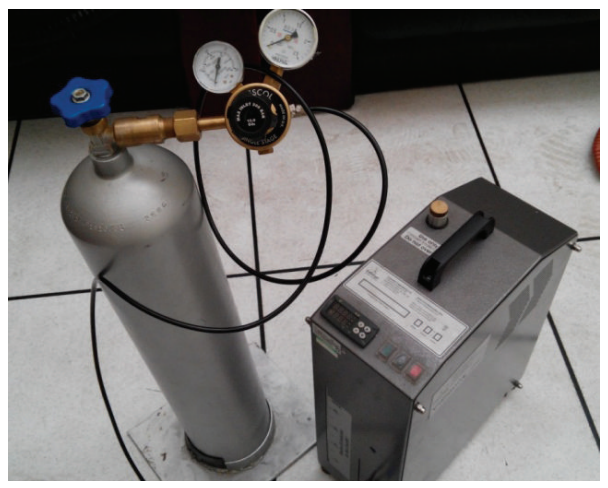

FIGURE 12: Smoke generator.

the same sections. This result is consistent with the relationship between the flow concentration and velocity as described in this paper.

Figure 5 shows that the area of the low-speed wake region of the square-back model was the largest, whereas that of the notchback model was the smallest. Thus, the exhaust gas of the square-back model had the largest diffusion region, and the diffusion region of the notchback model was the smallest and had the largest concentration gradient (Figure 10).

Propane diffusion mainly occurred in the body wake zone, as shown in Figure 10. The back view shows that the diffusion never extended beyond the body outline. The reason is that the airflow from the top and side of the body was faster than that in the wake region, as illustrated in Figures 6 and 7. These figures also demonstrate that the trace gas diffusion process was mainly affected by the wake flow field, but the concentration gradient was not.

4.3. Notchback MIRA Model Wind Tunnel Test. The automotive wind tunnel at Jilin University is an open reflux wind tunnel. Figure 11 shows the tunnel structure diagram.

The MIRA notchback 1:2 model was tested in the wind tunnel to examine the dispersion characteristics more intuitively. A Vicount 180 smoke generator system was used as the source of smoke, and a pipe was used to lead the smoke to the rear of the car. The diameter of the particles generated by the smoke generator was 0.2 microns to 0.3 microns. The smoke generator system is shown in Figure 12.

The model was placed directly in the test section. The model and placement are shown in Figure 13.

The exhaust pipe was fixed at $225 \mathrm{~mm}$ from the center line of the model and was parallel to the centerline during the test to be consistent with the exhaust pipe position in the numerical simulation. The actual vehicle exhaust pipe inner diameter was approximately $4 \mathrm{~cm}$; thus, the pipe diameter was approximately $2 \mathrm{~cm}$ in the test. Given the actual circumstances in the wind tunnel test section, the exhaust pipe was fixed at the right side of the model, as presented in Figure 14 (the model is shown backwards from the front).

Considering the experimental model for steel structures, we used the pressure measurement mainly to compare the flow field of the numerical simulation and verify the simulation accuracy. This test measured 26 pressure points on the body and center line of the rear body symmetry. Figure 15 illustrates the relative position of each pressure point.

The MIRA notchback 1:2 model was tested in the pressure test and flow pattern trial. The specific test program is presented in Table 1.

The pressure coefficients measured in the wind tunnel tests were $13 \mathrm{~m} / \mathrm{s}$ and $20 \mathrm{~m} / \mathrm{s}$, and the numerical simulation at 

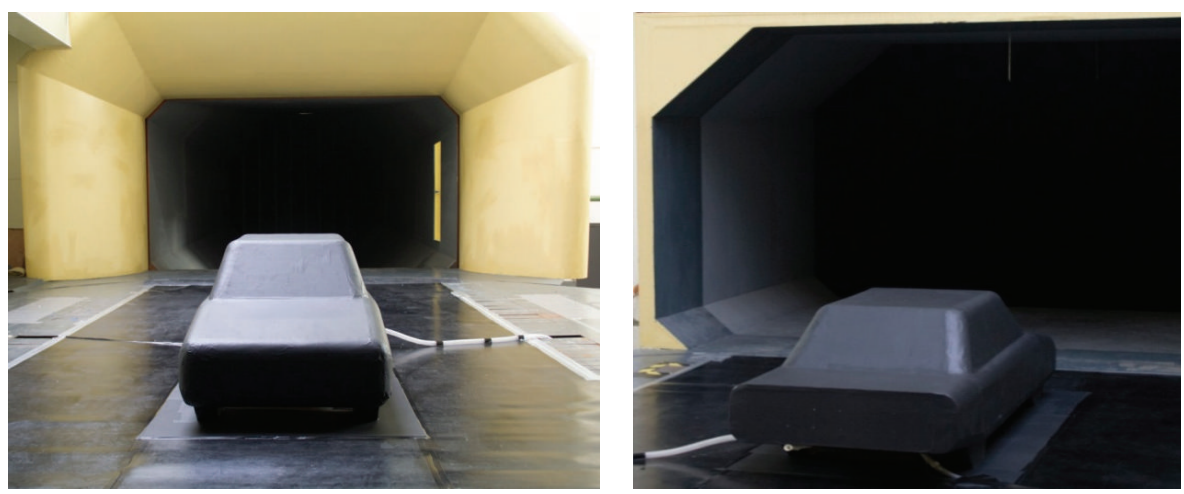

FIgURE 13: Model in placement test section.

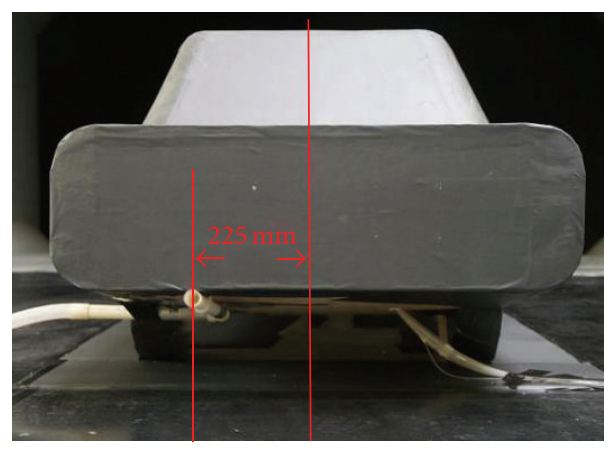

FIGURE 14: Exhaust pipe installation location.
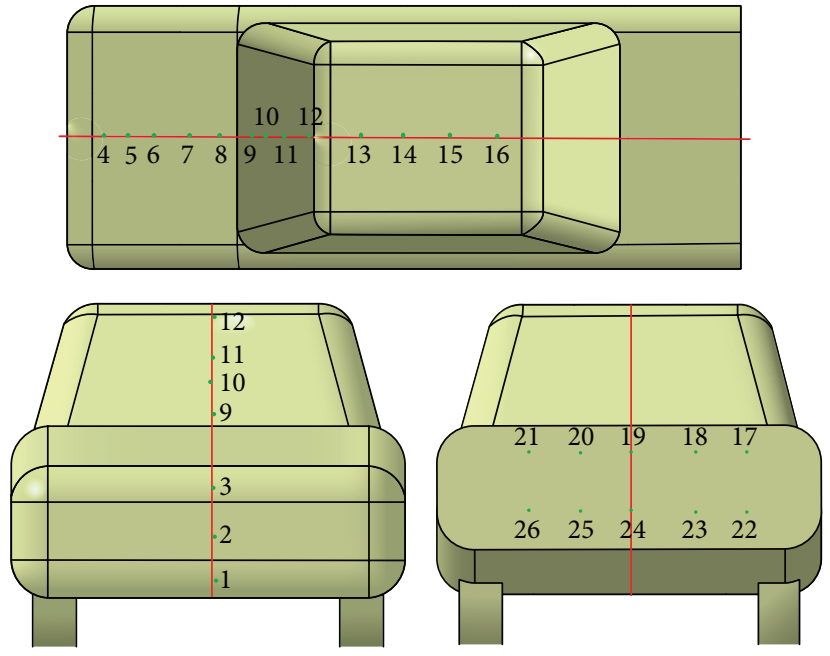

FIGURE 15: Schematic arrangement of measurement points.

$13 \mathrm{~m} / \mathrm{s}$ is shown in Figure 16. The pressure coefficient curves measured under the two test wind speeds were coincidental, and the differences were almost difficult to observe. The pressure coefficient trend in the numerical simulation was consistent with the experimental value. The pressure coefficients in the hood and windshield junction, as well as the windshield edges, were slightly different. This difference was caused by the improper fitting of the sludge structure with the wallpaper and a vortex between the wallpaper and model.
However, the pressure coefficient monitored in the tail almost coincided; this condition verifies the consistency of the wake field data between the test and numerical simulation to a certain extent.

Analyzing the numerical simulation results in the previous section shows that the underbody airflow exhibited an uptrend because of the notchback model tail inclination. The exhaust plume diffusion shows an upward trend because it was affected by the rising airflow. The plume was generated in 
TABLE 1: Specific test program of MIRA notchback 1:2 model.

\begin{tabular}{lccc}
\hline Experimental model & $\begin{array}{c}\text { Experimental } \\
\text { method }\end{array}$ & Wheel angle & Speed $(\mathrm{m} / \mathrm{s})$ \\
\hline MIRA notchback 1:2 & Pressure test & $0^{\circ}$ & 13,20 \\
model & Plume law & $0^{\circ}$ & 13 \\
\hline
\end{tabular}

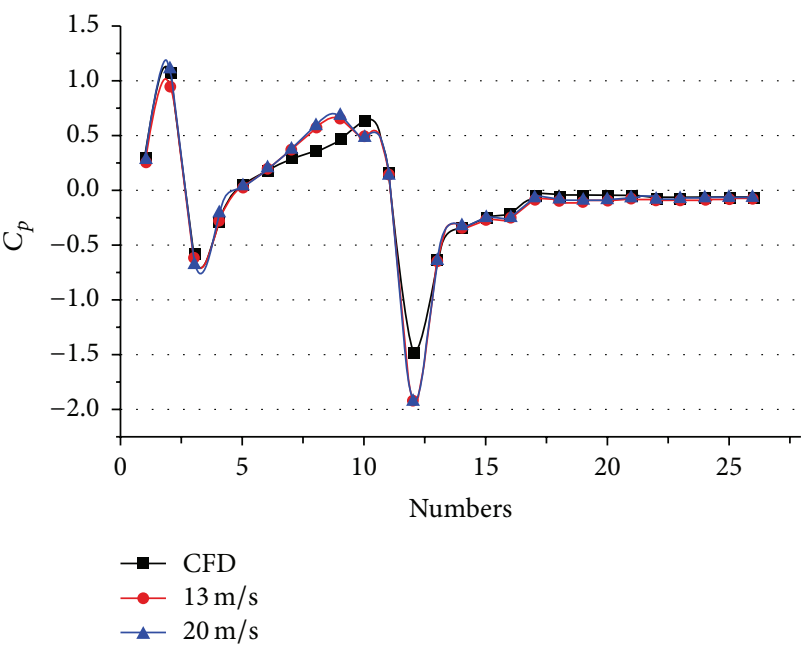

Figure 16: Pressure coefficient $\left(C_{p}\right)$ contrast curve.

the low-speed areas after being affected by the counterclockwise trailing vortex, as shown in Figure 17.

The plume diffusion region was mainly located in the automotive wake region. Given that the airflow speed that passes through the top and side of the body was higher than the tail, the exhaust gas diffusion region barely exceeded the outer contour of the body, as shown in Figure 18.

Another important feature of the plume diffusion in 3D space was that it spiraled backwards, as shown in Figure 19. The structure of the wake flow field was unobserved because of the limited test conditions. The characteristics were then analyzed using the numerical simulation results in the previous section.

The numerical simulation result of the velocity field in the three-section rear of the notchback car was analyzed. The three sections are $x / l=0.036, x / l=0.146$, and $x / l=$ 0.292 . Three cross-sections relative to the position of the vehicle are shown in Figure 20. The velocity fields in the three sections are presented in Figures 21 to 23. Yellow represents the velocity field of the high-speed zone and blue represents the low-speed zone.

Figures 21 to 23 illustrate that two reverse swirls occur in the rear of the car and the airflow moves backward after being affected by the high-velocity gas stream flowing outside the body and bottom (Figure 7). Therefore, the interaction of the two air flows resulted in a spiral trend for the flow. The analysis of the tracer-gas diffusion characteristics in the previous section shows that the diffusion was mainly influenced by the tail gas stream. Thus, the plume diffusion still spiraled backwards, as shown in Figure 19.

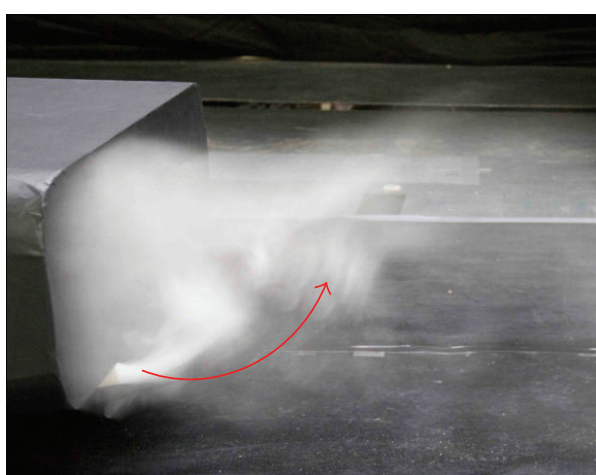

FIGURE 17: Uptrend trend.

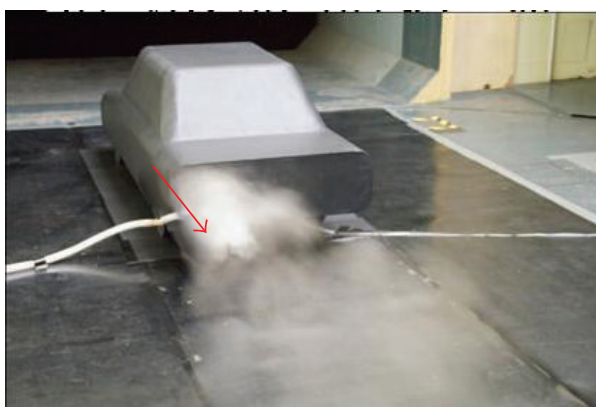

FIGURE 18: Near wake region diffusion profile.

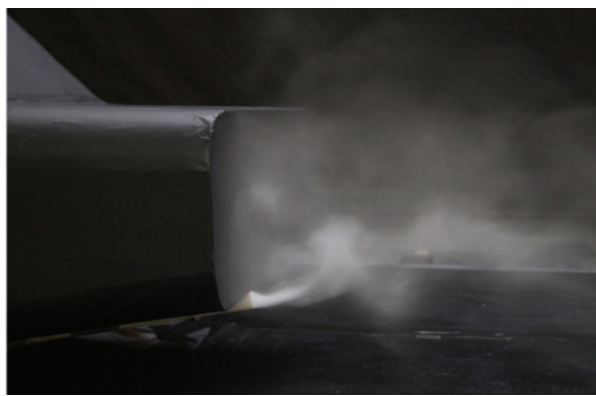

FIGURE 19: Spiral diffusion schematic.

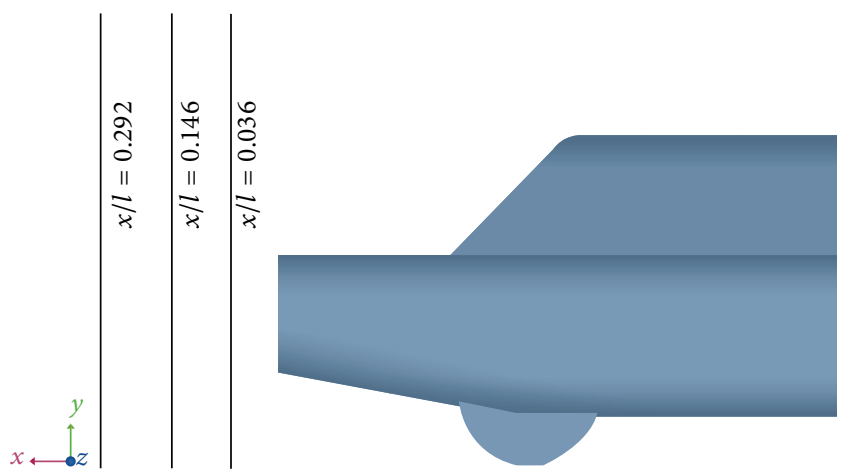

FIgURE 20: Relative positions of three sections. 


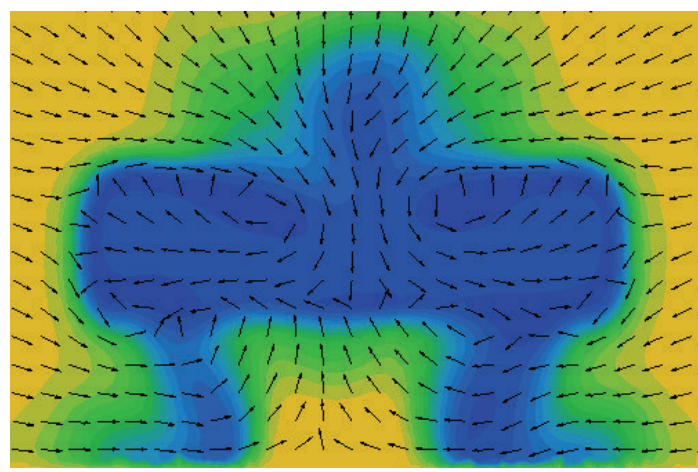

Figure 21: Velocity field of section $x / l=0.036$.

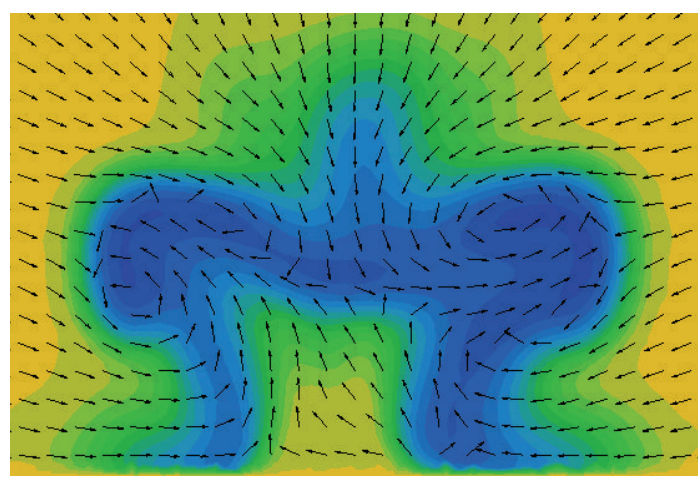

FIgURE 22: Velocity field of section $x / l=0.146$

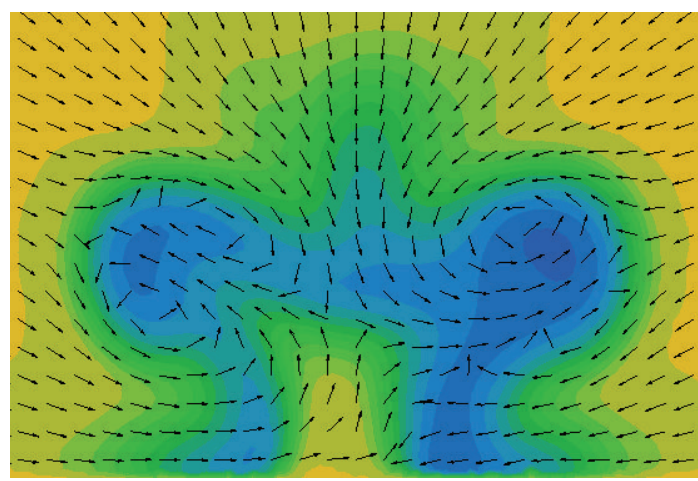

FIGURE 23: Velocity field of section $x / l=0.292$.

\section{Conclusions}

Three MIRA models were used to investigate exhaust gas diffusion. The exhaust gas diffusion process was mainly affected by the wake flow field and directly related to the velocity field. The exhaust gas spread upward in the $Y$ direction and outward in the $Z$ direction.

The wake area of the square-back model was larger and had an inclination on the tail, so the exhaust gas diffusion had the largest diffusion region in the wake field. The notchback had a minimum wake region, and the exhaust gas was involved in a trailing vortex because of the tail inclination. Thus, the diffusion region was the smallest. The exhaust gas for the fastback model, which had no tail inclination, was directly dragged into the wake region by a high-speed stream from the bottom of the body. The diffusion distance on the horizontal plane was also the longest.

\section{Conflict of Interests}

The authors declare no conflict of interests regarding the publication of this paper.

\section{Acknowledgments}

The authors are grateful for the support provided by the National Science Foundation of China (E50805062), the Key Laboratory of Road Construction Technology and Equipment (Changan University) of the Ministry of Education, the Scientific Frontier and Interdisciplinary Innovation Projects, and the Fundamental Research Funds for the Central Universities.

\section{References}

[1] Z.-Q. Yin, Research on the nanoparticle nucleation and coagulation processes in twin-jet exhaust plume of a vehicle [Ph.D. thesis], Zhejiang University, Hangzhou, China, 2008, (Chinese).

[2] Q. Zhang, Dispersion of Vehicle Exhaust Gas with Numerical Simulation CFD, Nanjing University of Science and Technology, Nanjing, China, 2007 (Chinese).

[3] L. Gidhagen, C. Johansson, J. Langner, and V. L. Foltescu, "Urban scale modeling of particle number concentration in Stockholm," Atmospheric Environment, vol. 39, no. 9, pp. 1711$1725,2005$.

[4] L. Isella, B. Giechaskiel, and Y. Drossinos, "Diesel-exhaust aerosol dynamics from the tailpipe to the dilution tunnel," Journal of Aerosol Science, vol. 39, no. 9, pp. 737-758, 2008.

[5] Z. Ning, C. S. Cheung, Y. Lu, M. A. Liu, and W. T. Hung, "Experimental and numerical study of the dispersion of motor vehicle pollutants under idle condition," Atmospheric Environment, vol. 39, no. 40, pp. 7880-7893, 2005.

[6] G. Dong and T. L. Chan, "Large eddy simulation of flow structures and pollutant dispersion in the near-wake region of a light-duty diesel vehicle," Atmospheric Environment, vol. 40, no. 6, pp. 1104-1116, 2006.

[7] I. Kanda, K. Uehara, Y. Yamao, Y. Yoshikawa, and T. Morikawa, "A wind-tunnel study on exhaust-gas dispersion from road vehicles-part II: effect of vehicle queues," Journal of Wind Engineering and Industrial Aerodynamics, vol. 94, no. 9, pp. 659673, 2006.

[8] T. L. Chan, D. D. Luo, C. S. Cheung, and C. K. Chan, "Large eddy simulation of flow structures and pollutant dispersion in the near-wake region of the studied ground vehicle for different driving conditions," Atmospheric Environment, vol. 42, no. 21, pp. 5317-5339, 2008.

[9] U. Uhrner, S. von Löwis, H. Vehkamäki et al., "Dilution and aerosol dynamics within a diesel car exhaust plume-CFD simulations of on-road measurement conditions," Atmospheric Environment, vol. 41, no. 35, pp. 7440-7461, 2007.

[10] B. Wehner, U. Uhrner, S. von Löwis, M. Zallinger, and A. Wiedensohler, "Aerosol number size distributions within the exhaust plume of a diesel and a gasoline passenger car under 
on-road conditions and determination of emission factors," Atmospheric Environment, vol. 43, no. 6, pp. 1235-1245, 2009.

[11] Y. J. Wang and K. M. Zhang, "Coupled turbulence and aerosol dynamics modeling of vehicle exhaust plumes using the CTAG model," Atmospheric Environment, vol. 59, pp. 284-293, 2012.

[12] K. Richards, Computational modelling of pollution dispersion in the near wake of a vehicle [Ph.D. thesis], University of Nottingham, Nottingham, UK, 2002.

[13] D.-H. Kim, M. Gautam, and D. Gera, "On the prediction of concentration variations in a dispersing heavy-duty truck exhaust plume using k- $\varepsilon$ turbulent closure," Atmospheric Environment, vol. 35, no. 31, pp. 5267-5275, 2001. 


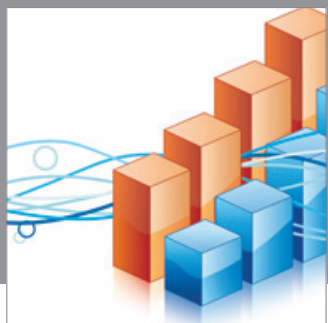

Advances in

Operations Research

mansans

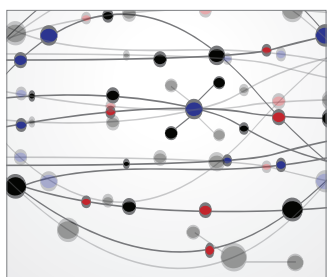

The Scientific World Journal
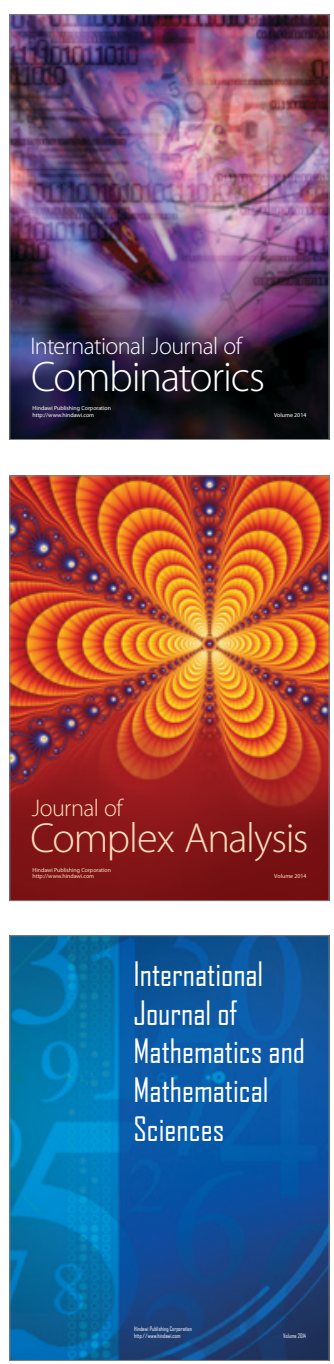
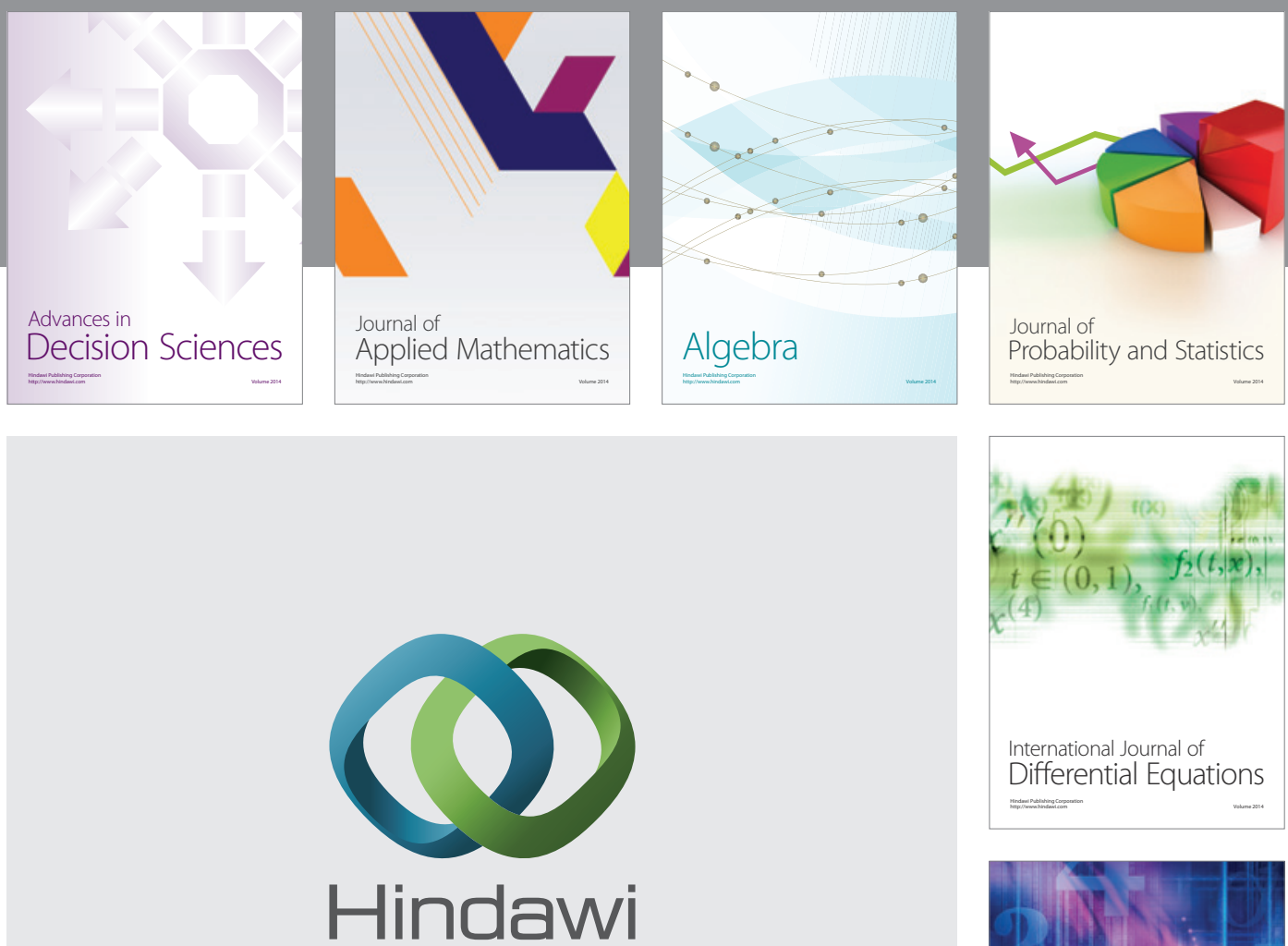

Submit your manuscripts at http://www.hindawi.com
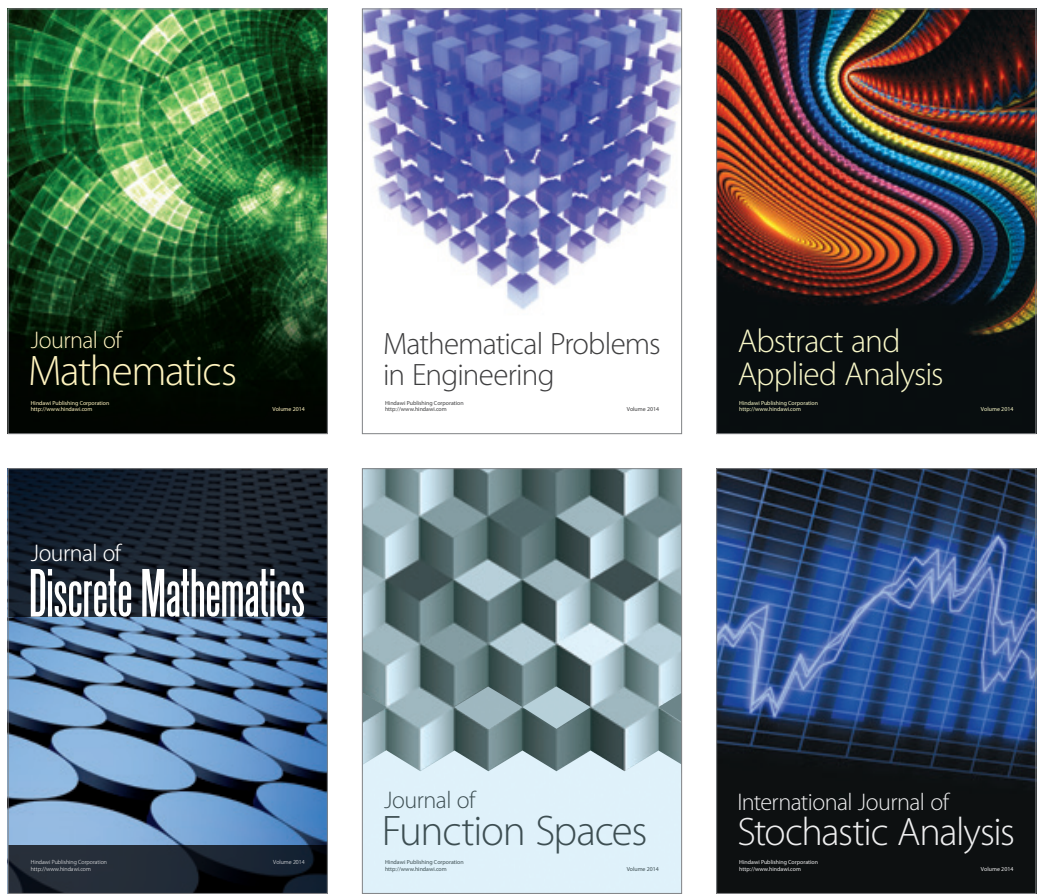

Journal of

Function Spaces

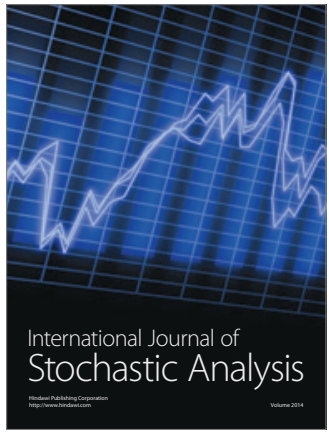

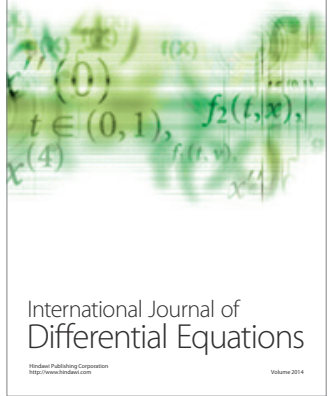
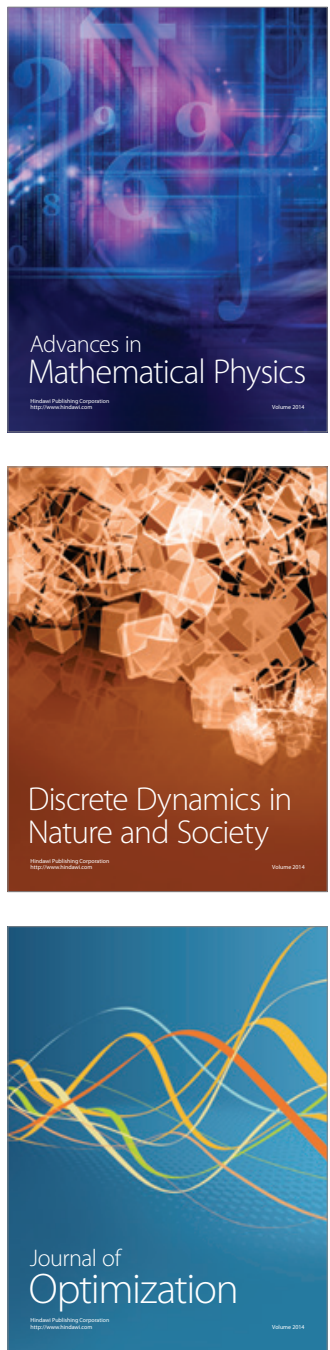